$62^{\text {ème }}$ Congrès de la SFCO, 02015 (2014)

DOI: $10.1051 /$ sfco/20146202015

(C) Owned by the authors, published by EDP Sciences, 2014

\title{
Le carcinome muco-épidermoïde du palais, prise en charge pluridisciplinaire : à propos d'un cas.
}

\author{
Bridonneau Th' ${ }^{1}$ Quinque $\mathrm{E}^{1}$, Zink $\mathrm{S}^{2}$, Debry $\mathrm{Ch}^{3}$, Bahi-Gross $\mathbf{S}^{1}$ \\ 1 CHRU de Strasbourg, Hôpital Civil, Unité Fonctionnelle de Chirurgie Buccale-Implantologie, 1 Place de l'Hôpital, 67000, Strasbourg, FRANCE \\ 2 CHRU de Strasbourg, Hôpital Civil, Service de Stomatologie, Chirurgie Maxillo-Faciale, Esthétique et Réparatrice, 1 place de l'Hôpital, 67000, Strasbourg, FRANCE \\ 3 CHRU de Strasbourg, Hôpital de Hautepierre, Service d'ORL et Chirurgie Cervico-Faciale, 1 Avenue de Molière, 67000, Strasbourg, FRANCE
}

Le carcinome muco-épidermoïde (CME) est une tumeur maligne des maxillaires qui représente 1 à $3 \%$ des cancers des voies aérodigestives supérieures (IARC, 2005) et $29 \%$ des tumeurs malignes des glandes salivaires (Ellis, Auclair, 1996). Le CME se développe à partir de toutes les glandes salivaires, le plus souvent au niveau de la glande parotide, mais peut également atteindre les glandes salivaires accessoires dans la partie postéro-latérale du palais. Majoritairement découvert entre 40 et 60 ans, des cas très précoces (4 ans) ont été décrit. (Belghiti et al, 2011). Les signes cliniques sont généralement peu évocateurs surtouts dans les stades initiaux. En fonction du type histologique découvert (de bas à haut grade), la thérapeutique nécessite une prise en charge chirurgicale associée ou non à une radiothérapie post-opératoire dans les cas de haut grade (Robert, Andrews, 2012).

Le cas d'une découverte fortuite d'un CME est rapporté chez une jeune fille de 18 ans adressée par son chirurgien-dentiste traitant pour les avulsions de ses dents de sagesse. La patiente ne révèle à l'anamnèse aucun antécédent médico-chirurgical spécifique.

L'examen endo-buccal met en évidence une macule violacée de $1,5 \mathrm{~cm}$ de diamètre au niveau du palais dur en regard des faces palatines des dents 26-27. Les limites sont nettes et la palpation est souple et indolore. L'orthopantomogramme ne révèle aucune image spécifique au niveau de cette zone.

L'aspect « bénin » de cette lésion a motivé la biopsie sans autres examens complémentaires préalables. L'anesthésie locale réalisée au niveau de la lésion laisse soudre un liquide visqueux jaunâtre révélateur d'une lésion d'une glande salivaire accessoire. L'examen anatomopathologique effectué sur un prélèvement de $0.8 \times 0.7 \mathrm{~cm}$ conclu à un CME bien différenciée de bas grade.
Un bilan d'extension comprenant un СВCT a permis de mettre en évidence une lésion nodulaire de $10 \mathrm{~mm}$ de large $\times 8 \mathrm{~mm}$ d'épaisseur sans aucune extension adjacente. La lésion est limitée à la zone osseuse circonscrite au tiers postérieur gauche de l'hémi-palais osseux. Un examen IRM et un scanner cervico-thoracique ont également complété ce bilan d'extension.

L'exérèse chirurgicale est réalisée par l'équipe d'ORL laissant une communication bucco-sinusienne de plus de $2 \mathrm{~cm}$ fermée par une prothèse obturatrice mise en place immédiatement en post-opératoire.

Après contrôle clinique et radiologique, à deux ans post-opératoire, la patiente est réadressée à l'équipe de chirurgie maxillo-faciale pour la reconstruction de la perte de substance maxillaire par un lambeau de rotation palatin.

Le CME est différent du carcinome épidermoïde, les facteurs de risque sont inconnus et la symptomatologie est fruste. Ce cas révèle qu'une lésion d'aspect clinique bénin peut cacher un processus malin qu'il est nécessaire de diagnostiquer précocement pour limiter les conséquences fonctionnelles inhérentes à l'intervention chirurgicale. II insiste également sur la nécessité d'organiser en aval du diagnostic une prise en charge pluridisciplinaire adaptée.

BRIDONNEAU Thomas thomas.bridonneau@gmail.com

This is an Open Access article distributed under the terms of the Creative Commons Attribution License 4.0, which permits unrestricted use, distribution, and reproduction in any medium, provided the original work is properly cited. 